\title{
Dispersion relation of anti-D meson in the Chiral Density Wave
}

\author{
Daiki Suenaga* \\ Nagoya University \\ E-mail: suenaqadhken.phys.nagova-u.ac.ip \\ Masayasu Harada \\ Nagoya University \\ E-mail: haradadhken.phys.nagova-u.ac.ip
}

\begin{abstract}
We summarize main points of our study on the dispersion relations for $\bar{D}$ mesons in the Chiral Density Wave (CDW) where the chiral symmetry is spontaneously broken by the inhomogeneous chiral condensate. Emplying the Bloch's theorem for $\bar{D}$ mesons wave function, we show that the collective modes appear, which have a negative group velocity for small momentum region. The minimum of the energy is realized when the momentum is equal to the wave number of the density wave.
\end{abstract}

Flavor Physics \& CP Violation 2015

May 25-29, 2015

Nagoya, Japan

* Speaker. 


\section{Introduction}

Sponenous chiral symmetry breaking is one of the most important structures of QCD in the low energy region. In the vacuum, the spontaneous chiral symmetry breaking is driven by the chiral condensate $\langle\bar{q} q\rangle \neq 0$, which is homogeneous. However, in the high density region, it was pointed [四] that the chiral symmetry can be broken by the inhomegenous chiral condensate, which we call the Chiral Density Wave (CDW) below.

In this write-up, we show main points of our work [] where we calculate the dispersion relations for $\bar{D}\left(0^{-}\right), \bar{D}^{*}\left(1^{-}\right), \bar{D}_{0}^{*}\left(0^{+}\right)$and $\bar{D}_{1}\left(1^{+}\right)$mesons in the CDW. In our work, in addition to the heavy quark symmetry, we adopt the chiral doubling structure among these anti-charmed mesons [B]], where the heavy quark doublet of $\left(\bar{D}, \bar{D}^{*}\right)$ mesons is the chiral partner to the one of $\left(\bar{D}_{0}^{*}, \bar{D}_{1}\right)$ mesons as the chiral partner to each other. In the chiral doubling strcuter, the mass difference of $\left(\bar{D}, \bar{D}^{*}\right)$ mesons and $\left(\bar{D}_{0}^{*}, \bar{D}_{1}\right)$ mesons comes from the chiral symmetry breaking, so that we can expect some changes of the dispersion relations for these anti-charmed mesons in the CDW which we collectively call the $\bar{D}$ mesons below. Since the potentials of the $\bar{D}$ mesons in this phase are periodic so that we employ the Bloch's theorem to get the dispersion relations. Our results show that there emerge collective modes which have a negative group velocity for small momentum region, and that the minimum of the energy of the collective modes is realized when the momentum is equal to the wave number of the density wave.

\section{Model}

We take into account $\left(\bar{D}, \bar{D}^{*}\right)=\left(0^{-}, 1^{-}\right)$doublet and $\left(\bar{D}_{0}^{*}, \bar{D}_{1}\right)=\left(0^{+}, 1^{+}\right)$doublet with regarding the chiral partner to each other in this write-up [B]. For light degrees, we include the $\sigma$ and $\pi$ through the conventional chiral field $M=\sigma+i \pi^{a} \tau^{a}$ with $\tau^{a}$ being the Pauli matrix. In the vacuum, this $M$ has a VEV as $\langle M\rangle=f_{\pi}$, where $f_{\pi}$ is the pion decay constant, $f_{\pi}=92.4 \mathrm{MeV}$. In our analysis, we use the following relativistic form of the Lagrangian for $\bar{D}$ mesons interacting with $M$ :

$$
\begin{aligned}
\mathscr{L}= & \partial_{\mu} \bar{D}_{0}^{*} \partial^{\mu} \bar{D}_{0}^{* \dagger}-m^{2} \bar{D}_{0}^{*} \bar{D}_{0}^{* \dagger}-\partial_{\mu} \bar{D}_{1 v} \partial^{\mu} \bar{D}_{1}^{\dagger v}+\partial_{\mu} \bar{D}_{1 v} \partial^{v} \bar{D}_{1}^{\dagger \mu}+m^{2} \bar{D}_{1 \mu} \bar{D}_{1}^{\dagger \mu} \\
& +\partial_{\mu} \bar{D} \partial^{\mu} \bar{D}^{\dagger}-m^{2} \bar{D} \bar{D}^{\dagger}-\partial_{\mu} \bar{D}_{v}^{*} \partial^{\mu} \bar{D}^{* \dagger v}+\partial_{\mu} \bar{D}_{v}^{*} \partial^{v} \bar{D}^{* \dagger \mu}+m^{2} \bar{D}_{\mu}^{*} \bar{D}^{* \dagger \mu} \\
& -\frac{1}{2} \frac{m \Delta_{m}}{f_{\pi}}\left[\bar{D}_{0}^{*}\left(M+M^{\dagger}\right) \bar{D}_{0}^{* \dagger}-\bar{D}_{1 \mu}\left(M+M^{\dagger}\right) \bar{D}_{1}^{\dagger \mu}-\bar{D}\left(M+M^{\dagger}\right) \bar{D}^{\dagger}+\bar{D}_{\mu}^{*}\left(M+M^{\dagger}\right) \bar{D}^{* \dagger \mu}\right] \\
& -\frac{1}{2} \frac{m \Delta_{m}}{f_{\pi}}\left[\bar{D}_{0}^{*}\left(M-M^{\dagger}\right) \bar{D}^{\dagger}-\bar{D}_{1 \mu}\left(M-M^{\dagger}\right) \bar{D}^{* \dagger \mu}-\bar{D}\left(M-M^{\dagger}\right) \bar{D}_{0}^{* \dagger}+\bar{D}_{\mu}^{*}\left(M-M^{\dagger}\right) \bar{D}_{1}^{\dagger \mu}\right],
\end{aligned}
$$

where we omit the terms including the derivative acting on the $M$ field. In the chiral dlubling structure, the mass difference between $\left(\bar{D}, \bar{D}^{*}\right)$ mesons and $\left(\bar{D}_{0}^{*}, \bar{D}_{1}\right)$ mesons comes from the spontaneous chiral symmetry due to the VEV of $M$, and then $m$ is regarded as the average mass and $\Delta_{m}$ as the mass difference, which are estimated experimentally as $m=2.19 \mathrm{GeV}$ and $\Delta_{m}=430 \mathrm{MeV}$, respectively.

Next, let us introduce the Chiral Density Wave (CDW) phase. In this phase, the classical solution of chiral field $M$ is expressed as [四]

$$
\langle M\rangle=\phi \cos (2 f x)+i \tau^{3} \phi \sin (2 f x) .
$$


where $x$ is the direction of density wave and $f$ is the wave number of this modulation, and $\phi$ measures the magnitude of chiral condensate. Note $f=0$ and $\phi=f_{\pi}$ corresponds to the vacuum configuration $\langle M\rangle=f_{\pi}$, while $\phi=0$ corresponds to the chiral symmetric phase $\langle M\rangle=0$. In the following, we shall show our results by omitting the derivative interactions with fixing $\phi$ to be $f_{\pi}$. The effects of derivative interactions as well as those by varying the value of $\phi$ are shown in Ref. [l]].

We take into account four mesons of $\bar{D}, \bar{D}^{*}, \bar{D}_{0}^{*}$ and $\bar{D}_{1}$, so that we have sixteen states, i.e., eight states of $\left(\bar{D}_{u}, \bar{D}_{u}^{* 1}, \bar{D}_{u}^{* 2}, \bar{D}_{u}^{* 3}, \bar{D}_{0 u}^{*}, \bar{D}_{1 u}^{1}, \bar{D}_{1 u}^{2}, \bar{D}_{1 u}^{3}\right)$, and eight states of $(u \leftrightarrow d)$ (We have used the convention that $\bar{D}_{u}$ is composed of an anti-charm quark and an up quark). These sixteen states are divided into eight sectors: $\left(\bar{D}_{u}, \bar{D}_{0 u}^{*}\right),\left(\bar{D}_{u}^{* 1}, \bar{D}_{1 u}^{1}\right),\left(\bar{D}_{u}^{* 2}, \bar{D}_{1 u}^{2}\right),\left(\bar{D}_{u}^{* 3}, \bar{D}_{1 u}^{3}\right)$, and $(u \leftrightarrow d)$. Two states in each sector mix with each other, while they do not mix with any states in other sectors. Thanks to the $Z_{2}$ symmetry shown in [四] together with another $Z_{2}$ symmetry for the spin flip of Brown Muck and the heavy quark symmetry, we can show that these eight sectors have the same dispersion relations. Then we consider only $\left(\bar{D}_{u}, \bar{D}_{0 u}^{*}\right)$ sector below.

Using variational method respect to $\bar{D}_{u}^{\dagger}$ and $\bar{D}_{0 u}^{* \dagger}$ states in the Lagrangian (2.1), and inserting the CDW configuration, we obtain the follwoing equations of motion (EoMs) in the presense of the CDW:

$$
\begin{aligned}
\left(\partial_{\mu} \partial^{\mu}+m^{2}\right) \bar{D}_{u}-m \Delta_{m} \cos (2 f x) \bar{D}_{u}+i m \Delta_{m} \sin (2 f x) \bar{D}_{0 u}^{*}=0 \\
\left(\partial_{\mu} \partial^{\mu}+m^{2}\right) \bar{D}_{0 u}^{*}+m \Delta_{m} \cos (2 f x) \bar{D}_{0 u}^{*}-i m \Delta_{m} \sin (2 f x) \bar{D}_{u}=0 .
\end{aligned}
$$

These EoMs contain the periodic potentials with the period of $a=\pi / f$ respect to $x$ axis reflecting the CDW.

\section{Results}

We solve the EoMs ([2.3) by employing the Bloch's theorem to get the energy eigenvalues of the system. In Fig. 且, we plot the dispersion relations for $\left(\bar{D}_{u}, \bar{D}_{0 u}^{*}\right)$ states for $f=100 \mathrm{MeV}$ (a), $f=200 \mathrm{MeV}$ (b), respectively. In each figure, the vertical axis shows the energy eigenvalue $E$ and the horizontal axis is for the momentum $q_{x}$ for $x$ direction, where we take the momenta for $y$ and $z$ directions to be zero, $q_{y}=q_{z}=0$. Four solid curves show our results, and for comparison we plot the dispersion relations for free $\bar{D}$ and $\bar{D}_{0}^{*}$ mesons using the dotted curves. The red and green solid curves increase with increasing momentum $q_{x}$. Since these two curves are close to the free dispersions shown by the dotted line, we think that these two modes are the medium modified $\left(\bar{D}_{u}\right.$, $\bar{D}_{0 u}^{*}$ ) states. On the other hand, the blue and purple curves once decrease and then increase. The decreasing energy leads to the negative velocity, which does not exist in the vacuum. This implies that these two modes shown by the blue and purple curves are the collective modes. Comparing Fig. I(a) and (b), we see that the minimum of the energy for the collective modes is realized for $q_{x}=f$.

\section{Summary}

We considered dispersion relations for $\bar{D}$ mesons in the CDW phase with regarding $\left(\bar{D}, \bar{D}^{*}\right)$ mesons and $\left(\bar{D}_{0}^{*}, \bar{D}_{1}\right)$ mesons as the chiral partner to each other. The potentials were periodic in this 


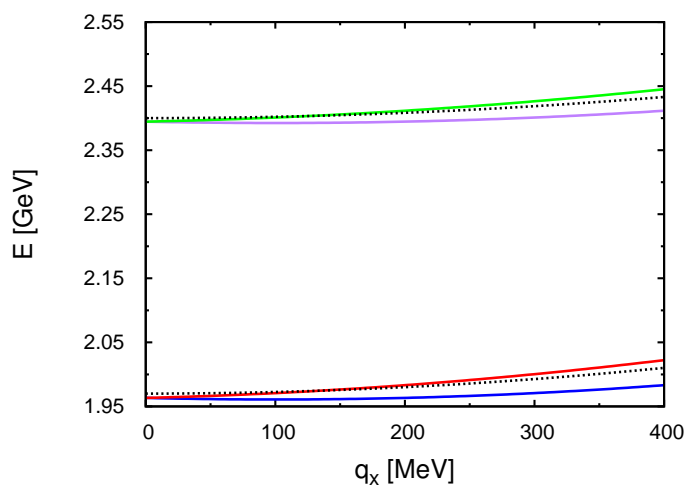

(a) $f=100 \mathrm{MeV}$

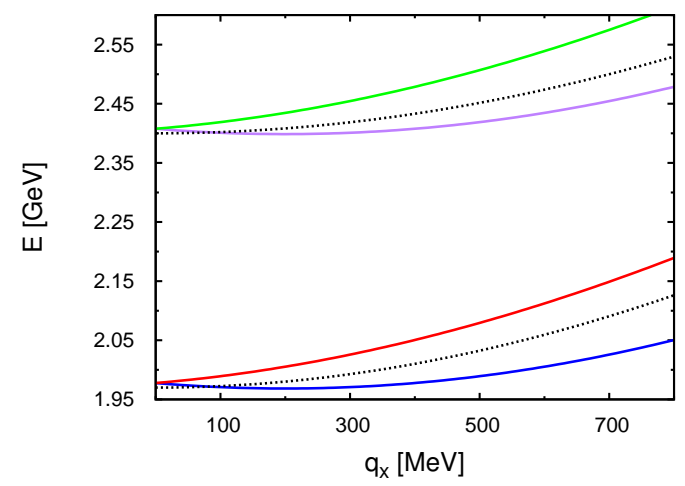

(b) $f=200 \mathrm{MeV}$

Figure 1: (color online) Dispersion relations $E$ vs $q_{x}$ for $f=100 \mathrm{MeV}$ (a) and $f=200 \mathrm{MeV}$ with $q_{x}=q_{y}=$ 0 . Solid curves show the results obtained in the present analysis, while the dotted curves are the dispersion relations for free $\bar{D}$ and $\bar{D}_{0}^{*}$ mesons, respectively.

phase so that we employed the Bloch's theorem to get the dispersion relations. Our result showed that the collective modes appeared, which had a negative group velocity for small momentum region. Furthermore, the minimum of the energy was realized when the momentum was equal to the wave number of the density wave. This may indicate the possibility of using the mesons including the heavy quark for probing the properties of high density medium.

In this write-up, we omitted the derivative interactions and the mass difference between $\bar{D}$ and $\bar{D}^{*}$ mesons which breaks the heavy quark symmetry. Furthermore, we fixed $\phi$ to be $f_{\pi}$, which can be varied to study the dependence on the value of the condensate. The results of these effects are shown in Ref. []].

\section{References}

[1] For example, E. Nakano and T. Tatsumi, Phys. Rev. D 71, 114006 (2005) [hep-ph/0411350]; D. Nickel, Phys. Rev. D 80, 074025 (2009) [arXiv:0906.5295 [hep-ph]]; T. Kojo, Y. Hidaka, L. McLerran and R. D. Pisarski, Nucl. Phys. A 843, 37 (2010) [arXiv:0912.3800 [hep-ph]]; M. Buballa and S. Carignano, Prog. Part. Nucl. Phys. 81, 39 (2015) [arXiv:1406.1367 [hep-ph]].

[2] D. Suenaga and M. Harada, in preparation.

[3] M. A. Nowak, M. Rho, and I. Zahed, Phys. Rev. D 48, 4370 (1993); W. A. Bardeen and C. T. Hill, Phys. Rev. D 49, 409 (1994).

[4] D. Suenaga, B. R. He, Y. L. Ma and M. Harada, Phys. Rev. C 89, no. 6, 068201 (2014) [arXiv:1403.5140 [hep-ph]]. 